\title{
Potential of edaravone for neuroprotection in neurologic diseases that do not involve cerebral infarction (Review)
}

\author{
KIYOSHI KIKUCHI ${ }^{1}$, KO-ICHI KAWAHARA ${ }^{2 *}$, HISAAKI UCHIKADO ${ }^{8 *}$, NAOHISA MIYAGI ${ }^{1}$, \\ TERUKAZU KURAMOTO ${ }^{9}$, TOMOYA MIYAGI ${ }^{9}$, YOKO MORIMOTO ${ }^{5}$, TAKASHI ITO ${ }^{2}$, \\ SALUNYA TANCHAROEN ${ }^{10}$, NAOKI MIURA $^{6}$, KAZUNORI TAKENOUCHI $^{2}$, YOKO OYAMA $^{2}$, \\ BINITA SHRESTHA $^{2}$, FUMIYO MATSUDA ${ }^{7}$, YOSHIHIRO YOSHIDA ${ }^{7}$, SHINIHIRO ARIMURA $^{3}$, \\ KENTARO MERA ${ }^{4}$, KO-ICHI TADA ${ }^{4}$, NARIMASA YOSHINAGA ${ }^{2}$, RYUICHI MAENOSONO $^{2}$, \\ YOSHIKO OHNO $^{2}$, TERUTO HASHIGUCHI ${ }^{2}$, IKURO MARUYAMA ${ }^{2}$ and MINORU SHIGEMORI ${ }^{8}$
}

${ }^{1}$ Department of Neurosurgery, Yame Public General Hospital, Yame 834-0034; ${ }^{2}$ Division of Laboratory and Vascular Medicine, Department of Advanced Therapeutics, Field of Cardiovascular and Respiratory Disorders;

${ }^{3}$ Department of Maxillofacial Diagnostic and Surgical Science, Field of Oral and Maxillofacial Rehabilitation; ${ }^{4}$ Department of Dermatology, Kagoshima University Graduate School of Medical and Dental Sciences, Kagoshima 890-8520, ${ }^{5}$ Department of Restorative Dentistry and Endodontology, Kagoshima University Graduate School of Medical and Dental Sciences, Kagoshima 890-8544; ${ }^{6}$ Veterinary Teaching Hospital and Laboratory of Veterinary Diagnostic Imaging, Faculty of Agriculture, Kagoshima University, Kagoshima 890-0065; ${ }^{7}$ Division of Physical Therapy, School of Health Sciences, Faculty of Medicine, Kagoshima University, Kagoshima 890-8506; ${ }^{8}$ Department of Neurosurgery, Kurume University School of Medicine, Kurume 830-0011; ${ }^{9}$ Department of Neurosurgery, Omuta City General Hospital, Fukuoka 836-8567, Japan; ${ }^{10}$ Department of Pharmacology, Faculty of Dentistry, Mahidol University, Bangkok 10400, Thailand

Received October 4, 2010; Accepted May 19, 2011

DOI: $10.3892 / \mathrm{etm} .2011 .281$

\begin{abstract}
Edaravone was originally developed as a potent free radical scavenger and has been widely used to treat cerebral infarction in Japan since 2001. Several free radical scavengers have been developed and some of them have progressed to clinical trials for the treatment of cerebral infarction. One such scavenger, edaravone, has been approved by the regulatory authority in Japan for the treatment of patients with cerebral infarction. Of particular interest is the ability of edaravone to diffuse into the central nervous system in various neurologic diseases. Aside from its hydroxyl radical scavenging effect, edaravone has been found to have beneficial effects on inflammation, matrix metalloproteinases, nitric oxide production and
\end{abstract}

Correspondence to: Dr Ko-ichi Kawahara, Division of Laboratory and Vascular Medicine, Field of Cardiovascular and Respiratory Disorders, Department of Advanced Therapeutics, Kagoshima University Graduate School of Medical and Dental Sciences, 8-35-1 Sakuragaoka, Kagoshima 890-8520, Japan

E-mail: telo@m3.kufm.kagoshima-u.ac.jp

${ }^{*}$ Contributed equally

Key words: edaravone, cerebral infarction, free radical scavenger, neuroprotection, neurologic disease apoptotic cell death. Concordantly, edaravone has been found to have neuroprotective effects in a number of animal models of disease, including stroke, spinal cord injury, traumatic brain injury, neurodegenerative diseases and brain tumors. The proven safety of edaravone following 9 years of use as a free radical scavenger suggests that it may have potential for development into an effective treatment of multiple neurologic conditions in humans.

\section{Contents}

1. Introduction

2. Effects of edaravone on various neurologic diseases that do not involve cerebral infarction

3. Current and future developments

\section{Introduction}

Edaravone (Mitsubishi Tanabe Pharma Corporation, Tokyo, Japan) was the first neuroprotective drug to be introduced worldwide and, since 2001, has been used to treat numerous patients with cerebral infarction in Japan. Edaravone has also been introduced in the US for the early management of adults with ischemic stroke (1-3). The anti-oxidant actions of edaravone are believed to enhance prostacyclin production, inhibit lipoxygenase metabolism of arachidonic acid by trapping hydroxyl radicals, inhibit alloxan-induced lipid peroxidation 
and quench active oxygen. These effects lead to the protection of various cell types, such as endothelial and myocardial cells, against damage by reactive oxygen species (ROS) (4). Although many compounds have been evaluated, few drugs have been found to be successful in studies conducted in Western countries. By contrast, trials conducted by Japanese researchers have been more successful (5). Several free radical scavengers have been developed and certain of these, including ebselen, tirilazad and NXY-059, have progressed to clinical trials (6). However, trials of ebselen and tirilazad in patients with cerebral infarction were terminated because of inadequate therapeutic effects $(7,8)$. Meanwhile, in the Stroke-Acute Ischemic NXY Treatment II trials, NXY-059 was found to be ineffective for the treatment of cerebral infarction when administered within $6 \mathrm{~h}$ after the onset of symptoms (9).

Recently, edaravone has been found to have other properties unrelated to its anti-oxidant actions that may be useful for the treatment of a number of other diseases (Table I). Of particular interest is the potential for the use of edaravone in neurologic conditions, such as subarachnoid hemorrhage (SAH), intracerebral hemorrhage (ICH), spinal cord injury (SCI), traumatic brain injury (TBI), amyotrophic lateral sclerosis (ALS) and Parkinson's disease (PD). To the best of our knowledge, no reports have reviewed a role for edaravone in various neurologic diseases that do not involve cerebral infarction to date. The purpose of this review is to examine the properties of edaravone that may lead to neuroprotection and to summarize the current state of research on the use of edaravone in a number of animal models of neurologic disease.

One clinical trial found that the administration of edaravone alone within $72 \mathrm{~h}$ of cerebral infarction onset significantly reduced infarct volume and produced sustained benefits during a 3-month follow-up period $(10,11)$. Recently, Unno et al reported that the total amount of edaravone used was associated with its efficacy in terms of rehabilitation gain (12). Meanwhile, the administration of edaravone within $24 \mathrm{~h}$ of the onset of cerebral infarction was used for patients with lacunae, large-artery atherosclerosis and cardioembolism cerebral infarctions (2). Several lines of evidence have shown that edaravone has neuroprotective effects following brain injury after cerebral infarction. For example, cerebral infarction is associated with enhanced expression of aquaporin-4 (AQP4), which causes acute edema, metalloproteinase-9 (MMP-9) and the release of high-mobility group box-1 (HMGB-1) from affected tissue, worsening the clinical outcomes $(2,3,5,13,14)$. Edaravone is a low-molecular-weight agent that readily crosses the blood-brain barrier and its activity is not limited to the vascular compartment $(4,15)$. Furthermore, edaravone was found to inhibit MMP-9-related brain hemorrhage in rats treated with recombinant tissue plasminogen activator (14) and attenuate cerebral ischemic injury by suppressing AQP-4 (3). Moreover, edaravone was found to rescue rats from cerebral infarction by attenuating the release of HMGB-1 in neuronal cells (2). Taken together, these findings suggest that edaravone may be used to treat patients with cerebral infarction by targeting and inhibiting the deleterious molecular events associated with brain injury.

\section{Effects of edaravone on various neurologic diseases that do not involve cerebral infarction}

Subarachnoid hemorrhage. A SAH caused by rupture of a saccular cerebral aneurysm (CA) is a life-threatening disease with a 30-day mortality rate of $45 \%$ and mild-to-severe morbidity rate of $30 \%(16,17)$. CA is a relatively common disease with a prevalence ranging from 1 to $5 \%$ as found in a large autopsy series (18). Despite its clinical and public significance, the detailed mechanisms of the initiation, progression and rupture of CAs remain to be elucidated, resulting in the absence of effective medical treatment for patients with ruptured and unruptured CAs (19). Yet, edaravone has been found to effectively inhibit CA formation by suppressing inflammation-related gene expression in aneurysmal walls in mice (19).

Cerebral vasospasm is one of the major causes of mortality and morbidity in patients with SAH (20). The detailed pathogenesis of cerebral vasospasm remains unclear and, in spite of intense and extensive investigations over the past four decades, no optimal treatment has been established (21). Recently, Munakata et al reported a trend toward a lesser incidence of delayed ischemic neurological deficits and a lesser incidence of poor outcome caused by cerebral vasospasm in edaravone-treated patients (22). Edaravone was found to exhibit a clear and selective inhibitory effect against hydroxyl radical-induced vasocontraction in the canine basilar artery in vitro (23).

Intracerebral hemorrhage. ICH is an often-fatal stroke subtype (24) and accounts for 8-15\% of all strokes in Western populations and $20-30 \%$ in Asian populations (25). ICH frequently produces severe neurologic deficits due to secondary brain edema (24). Edaravone was found to attenuate ICH-induced brain edema, neurologic deficits and oxidative injury in rats. In addition, edaravone was found to reduce iron- and thrombininduced brain injury (24).

Ischemic spinal cord injury. One of the most serious clinical diseases is SCI, the incidence of which has been increasing yearly (26). Spinal cord repair is a problem that has long puzzled neuroscientists $(27,28)$. The repair of the injured human spinal cord with resultant functional recovery is one of the major challenges of contemporary neuroscience (29). Although the mortality rate of SCI has dropped to $<5 \%$, the disability rate associated with SCI remains high (26). Edaravone has a protective effect on spinal cord neurons, both neurologically and histologically, by suppressing the level of free radical species in rabbits $(30,31)$. Edaravone prevents spinal cord damage and affects the enzyme levels of nitric oxide synthase and $\mathrm{Cu} / \mathrm{Zn}$ superoxide dismutase (SOD) after transient ischemia in rabbits (32). Furthermore, edaravone was demonstrated to reduce oxidative cellular damage and to increase DNA repair function in the rabbit spinal cord after transient ischemia (33). Moreover, evaluation of the effect of edaravone on lipid peroxide formation and downstream of the cascade of ROS production by measuring malonyldialdehyde in injured spinal cord homogenates found that edaravone significantly attenuated lipid peroxide formation by $>45 \%$ in the acute stage of SCI (34). 
Table I. Beneficial effects of edaravone in models of neurologic disease.

\begin{tabular}{|c|c|c|c|}
\hline Model & Effect of edaravone & Subjects & Refs. \\
\hline Cerebral aneurysm & Anti-inflammatory & Mice & 19 \\
\hline Cerebral vasospasm & Scavenges hydroxyl radicals & Cells & 23 \\
\hline Intracerebral hemorrhage & Reduces iron- and thrombin-induced brain injury & Rats & 24 \\
\hline \multicolumn{4}{|l|}{ Spinal cord injury } \\
\hline & Decreases nNOS & Rabbits & 32 \\
\hline & Increases eNOS & Rabbits & 32 \\
\hline & Increases $\mathrm{Cu} / \mathrm{Zn} \mathrm{SOD}$ & Rabbits & 32 \\
\hline & Increases DNA repair function & Rabbits & 33 \\
\hline \multicolumn{4}{|l|}{ Traumatic brain injury } \\
\hline & Anti-apoptotic & Rats & 39 \\
\hline & Increase neural stem cells & Rats & 40 \\
\hline & Decreases alkoxyl radicals & Patients, rats & 41,42 \\
\hline \multicolumn{4}{|c|}{ Amyotrophic lateral sclerosis } \\
\hline & Decreases SOD1 deposition & Mice & 46 \\
\hline & Anti-oxidant & Patients & 49 \\
\hline \multicolumn{4}{|l|}{ Parkinson's disease } \\
\hline & Anti-apoptotic & Rats & 52 \\
\hline & Anti-oxidant & Rats & 52 \\
\hline & Anti-inflammatory & Rats & 52 \\
\hline Multiple sclerosis & Decreases iNOS & Mice & 55 \\
\hline Radiation injury & Anti-apoptotic & Cells, mice & 56,60 \\
\hline
\end{tabular}

nNOS, neuronal nitric oxide synthase; eNOS, endothelial nitric oxide synthase; SOD, superoxide dismutase.

Traumatic brain injury. TBI presents a major worldwide social, economic and health problem (35). In the US, the mortality rate is estimated to be $21 \% 30$ days after TBI (36). TBI occurs as a result of a direct mechanical insult to the brain and induces central nervous system degeneration and neuronal cell death $(37,38)$. Edaravone administration following TBI was found to inhibit free radical-induced neuronal degeneration and apoptotic cell death around the damaged area, and to improve cerebral dysfunction following TBI in rats (39). Furthermore, edaravone increased neural stem cell numbers around the area of damage following rat TBI (40). Moreover, edaravone scavenges alkoxyl radicals in both patients and rats with TBI $(41,42)$.

Amyotrophic lateral sclerosis. ALS is one of the most common neuromuscular diseases with a worldwide incidence of 8 cases per 100,000 individuals per year (43). ALS is a devastating neurodegenerative disorder characterized by progressive and relatively selective degeneration of upper and lower motor neurons (44). Patients suffer from atrophy and paralysis of systemic voluntary muscles, including respiratory muscles, leading to respiratory failure and subsequent death 3-5 years after disease onset (44). An effective therapy for ALS that is capable of ameliorating its clinical course remains unknown (44). Twenty percent of familial ALS is caused by mutations in the $\mathrm{Cu} / \mathrm{Zn}$-dependent SOD1 gene, which was first reported in 1993 (45). The deposition of abnormal SOD1 in the anterior horns was found to be reduced by edaravone administration
(46). Since the administration of edaravone was found to result in a marked decrease in the 3-nitrotyrosine/tyrosine ratio, a marker of oxidative stress, the suppression of oxidative stress is likely to be upstream of the inhibition of aggregate formation (46-48). Furthermore, edaravone was found to effectively slow symptom progression and motor neuron degeneration in a mouse model of ALS (46). The beneficial effects of edaravone on Wobbler mice with ALS-like symptoms have also been reported (49). Moreover, a small-sized open trial with edaravone suggested that the drug is safe and may delay the progression of functional motor disturbances in ALS patients (49). Yoshino and Kimura reported that the concentration of 3-nitrotyrosine in the cerebrospinal fluid from ALS patients was reduced following intravenous administration of edaravone (49). Thus, edaravone is a promising therapeutic agent for human motor neuron diseases, including ALS (50). However, evidence for the beneficial effects of edaravone on human ALS patients awaits the publication of the results of a phase III clinical trial of ALS currently ongoing in Japan (50).

Parkinson's disease. PD is the most common neurodegenerative disorder after Alzheimer's disease (51). The prevalence is estimated at $0.3 \%$ in the entire population in industrialized countries, rising to $1 \%$ in those over 60 years of age and to $4 \%$ of the population over 80 (51). Studies regarding its incidence report that it is between 8 and 18 per 100,000 individuals per year (51). Edaravone was found to exert neuroprotective effects on PD models both in vivo (52) and in vitro $(52,53)$. 
The underlying mechanisms may involve anti-apoptotic, anti-oxidative and/or the anti-inflammatory effects of edaravone $(52,53)$. Edaravone may be a potential therapeutic agent for $\mathrm{PD}$, although the high therapeutic dosage required is a problem for clinical applications $(52,53)$.

Multiple sclerosis. Multiple sclerosis (MS) has a prevalence that ranges between 2 and 150 per 100,000 individuals depending on the country or specific population (54). Research directions regarding MS treatments include investigations aimed to better understand its pathogenesis and heterogeneity; research of more effective, convenient or tolerable new treatments; creation of therapies for progressive subtypes; neuroprotection strategies and the search for effective symptomatic treatments (55).

Brain tumors. Cranial radiation therapy is widely used to treat primary and metastatic brain tumors and head and neck cancers (56). Patients who receive radiotherapy involving the brain frequently experience progressive cognitive decline $(57,58)$. White matter necrosis and vasculopathy are overt pathologies caused by radiation-induced injury (59). Edaravone was found to protect human neural stem cells from radiationinduced apoptosis (60). In mouse models, edaravone protected neurons from apoptosis after irradiation and protected spatial memory retention deficits (56).

\section{Current and future developments}

In this review, we report the possible beneficial effects of edaravone, not only on brain injury following ischemia and reperfusion in patients with cerebral infarction, but also on various neurologic diseases. Recently, it has been reported that edaravone is effective in various models of neurologic disease (Table I). Due to the lack of clinical studies using edaravone, it remains unclear whether edaravone treatment is beneficial for patients who have excess oxidative stress and whether edaravone reduces the mortality rate of these patients. Controlled studies using a large population of patients are required to determine the effects of edaravone on various diseases. It is expected that edaravone may be useful for the treatment of various diseases in which oxidative stress may be involved in the pathogenesis.

\section{References}

1. Adams HP Jr, del Zoppo G, Alberts MJ, et al: Guidelines for the early management of adults with ischemic stroke: a guideline from the American Heart Association/American Stroke Association Stroke Council, Clinical Cardiology Council, Cardiovascular Radiology and Intervention Council, and the Atherosclerotic Peripheral Vascular Disease and Quality of Care Outcomes in Research Interdisciplinary Working Groups: the American Academy of Neurology affirms the value of this guideline as an educational tool for neurologists. Stroke 38: 1655-1711, 2007.

2. Kikuchi K, Kawahara K, Tancharoen S, et al: The free radical scavenger edaravone rescues rats from cerebral infarction by attenuating the release of high-mobility group box-1 in neuronal cells. J Pharmacol Exp Ther 329: 865-874, 2009.

3. Kikuchi K, Tancharoen S, Matsuda F, et al: Edaravone attenuates cerebral ischemic injury by suppressing aquaporin-4. Biochem Biophys Res Commun 390: 1121-1125, 2009.

4. Higashi Y, Jitsuiki D, Chayama K and Yoshizumi M: Edaravone (3-methyl-1-phenyl-2-pyrazolin-5-one), a novel free radical scavenger for treatment of cardiovascular diseases. Recent Pat Cardiovasc Drug Discov 1: 85-93, 2006.
5. Lapchak PA and Zivin JA: The lipophilic multifunctional antioxidant edaravone (Radicut) improves behavior following embolic strokes in rabbits: a combination therapy study with tissue plasminogen activator. Exp Neurol 215: 95-100, 2009.

6. Wang CX and Shuaib A: Neuroprotective effects of free radical scavengers in stroke. Drugs Aging 24: 537-546, 2007.

7. Green AR and Shuaib A: Therapeutic strategies for the treatment of stroke. Drug Discov Today 11: 681-693, 2006.

8. Van der Worp HB, Kappelle LJ, Algra A, et al: The effect of tirilazad mesylate on infarct volume of patients with acute ischemic stroke. Neurology 58: 133-135, 2002.

9. Shuaib A, Lees KR, Lyden P, et al: NXY-059 for the treatment of acute ischemic stroke. N Engl J Med 357: 562-571, 2007.

10. Edaravone Acute Infarction Study Group: Effect of a novel free radical scavenger, edaravone (MCI-186), on acute brain infarction. Randomized, placebo-controlled, double-blind study at multicenters. Cerebrovasc Dis 15: 222-229, 2003.

11. Zhang N, Komine-Kobayashi M, Tanaka R, Liu M, Mizuno Y and Urabe T: Edaravone reduces early accumulation of oxidative products and sequential inflammatory responses after transient focal ischemia in mice brain. Stroke 36: 2220-2225, 2005.

12. Unno Y, Katayama M and Shimizu H: Does functional outcome in acute ischaemic stroke patients correlate with the amount of free-radical scavenger treatment? A retrospective study of edaravone therapy. Clin Drug Investig 30: 143-155, 2010.

13. Papadopoulos MC, Krishna S and Verkman AS: Aquaporin water channels and brain edema. Mt Sinai J Med 69: 242-248, 2002.

14. Yagi K, Kitazato KT, Uno M, et al: Edaravone, a free radical scavenger, inhibits MMP-9-related brain hemorrhage in rats treated with tissue plasminogen activator. Stroke 40: 626-631, 2009.

15. Yoshida H, Yanai H, Namiki Y, Fukatsu-Sasaki K, Furutani N and Tada N: Neuroprotective effects of edaravone: a novel free radical scavenger in cerebrovascular injury. CNS Drug Rev 12: 9-20, 2006.

16. Feigin VL and Findlay M: Advances in subarachnoid hemorrhage. Stroke 37: 305-308, 2006

17. Van Gijn J, Kerr RS and Rinkel GJ: Subarachnoid haemorrhage. Lancet 369: 306-318, 2007.

18. Wiebers DO, Whisnant JP, Huston J III, et al: Unruptured intracranial aneurysms: natural history, clinical outcome, and risks of surgical and endovascular treatment. Lancet 362: 103-110, 2003.

19. Aoki T, Nishimura M, Kataoka H, Ishibashi R, Nozaki K and Hashimoto N: Reactive oxygen species modulate growth of cerebral aneurysms: a study using the free radical scavenger edaravone and p47phox(-/-) mice. Lab Invest 89: 730-741, 2009.

20. Dorsch NW and King MT: A review of cerebral vasospasm in aneurysmal subarachnoid haemorrhage Part I: incidence and effects. J Clin Neurosci 1: 19-26, 1994.

21. Nakagomi T, Yamakawa K, Sasaki T, Saito I and Takakura K: Effect of edaravone on cerebral vasospasm following experimental subarachnoid hemorrhage. J Stroke Cerebrovasc Dis 12: 17-21, 2003.

22. Munakata A, Ohkuma H, Nakano T, Shimamura N, Asano K and Naraoka M: Effect of a free radical scavenger, edaravone, in the treatment of patients with aneurysmal subarachnoid hemorrhage. Neurosurgery 64: 423-429, 2009.

23. Tosaka M, Hashiba Y, Saito N, Imai H, Shimizu T and Sasaki T: Contractile responses to reactive oxygen species in the canine basilar artery in vitro: selective inhibitory effect of MCI-186, a new hydroxyl radical scavenger. Acta Neurochir 144: 1305-1310, 2002.

24. Nakamura T, Kuroda Y, Yamashita S, et al: Edaravone attenuates brain edema and neurologic deficits in a rat model of acute intracerebral hemorrhage. Stroke 39: 463-469, 2008.

25. Fayad PB and Awad IA: Surgery for intracerebral hemorrhage. Neurology 51: S69-S73, 1998.

26. McDonald JW and Sadowsky C: Spinal-cord injury. Lancet 359: 417-425, 2002.

27. Andersson U, Wang H, Palmblad K, et al: High mobility group 1 protein (HMG-1) stimulates proinflammatory cytokine synthesis in human monocytes. J Exp Med 192: 565-570, 2000.

28. Ao Q, Wang AJ, Chen GQ, Wang SJ, Zuo HC and Zhang XF: Combined transplantation of neural stem cells and olfactory ensheathing cells for the repair of spinal cord injuries. Med Hypotheses 69: 1234-1237, 2007.

29. Rosenfeld JV, Bandopadhayay P, Goldschlager T and Brown DJ: The ethics of the treatment of spinal cord injury: stem cell transplants, motor neuroprosthetics, and social equity. Top Spinal Cord Inj Rehabil 14: 76-88, 2008. 
30. Aoyama T, Hida K, Kuroda S, et al: Edaravone (MCI-186) scavenges reactive oxygen species and ameliorates tissue damage in the murine spinal cord injury model. Neurol Med Chir 48: 539-545, 2008

31. Suzuki K, Kazui T, Terada H, et al: Experimental study on the protective effects of edaravone against ischemic spinal cord injury. J Thorac Cardiovasc Surg 130: 1586-1592, 2005.

32. Takahashi G, Sakurai M, Abe K, Itoyama Y and Tabayashi K: MCI-186 prevents spinal cord damage and affects enzyme levels of nitric oxide synthase and $\mathrm{Cu} / \mathrm{Zn}$ superoxide dismutase after transient ischemia in rabbits. J Thorac Cardiovasc Surg 126: 1461-1466, 2003.

33. Takahashi G, Sakurai M, Abe K, Itoyama Y and Tabayashi K: MCI-186 reduces oxidative cellular damage and increases DNA repair function in the rabbit spinal cord after transient ischemia. Ann Thorac Surg 78: 602-607, 2004.

34. Ohta S, Iwashita Y, Takada H, Kuno S and Nakamura T: Neuroprotection and enhanced recovery with edaravone after acute spinal cord injury in rats. Spine 30: 1154-1158, 2005.

35. Maas AI, Stocchetti N and Bullock R: Moderate and severe traumatic brain injury in adults. Lancet Neurol 7: 728-741, 2008

36. Greenwald BD, Burnett DM and Miller MA: Congenital and acquired brain injury. 1. Brain injury: epidemiology and pathophysiology. Arch Phys Med Rehabil 84: S3-S7, 2003.

37. Chirumamilla S, Sun D, Bullock MR and Colello RJ: Traumatic brain injury induced cell proliferation in the adult mammalian central nervous system. J Neurotrauma 19: 693-703, 2002.

38. Rice AC, Khaldi A, Harvey HB, et al: Proliferation and neuronal differentiation of mitotically active cells following traumatic brain injury. Exp Neurol 183: 406-417, 2003.

39. Itoh T, Satou T, Nishida S, et al: Edaravone protects against apoptotic neuronal cell death and improves cerebral function after traumatic brain injury in rats. Neurochem Res 35: 348-355, 2009.

40. Itoh T, Satou T, Nishida S, Tsubaki M, Hashimoto S and Ito H: The novel free radical scavenger, edaravone, increases neural stem cell number around the area of damage following rat traumatic brain injury. Neurotox Res 16: 378-389, 2009.

41. Dohi K, Satoh K, Mihara Y, et al: Alkoxyl radical-scavenging activity of edaravone in patients with traumatic brain injury. J Neurotrauma 23: 1591-1599, 2006

42. Dohi K, Satoh K, Nakamachi T, et al: Does edaravone (MCI$186)$ act as an antioxidant and a neuroprotector in experimental traumatic brain injury? Antioxid Redox Signal 9: 281-287, 2007.

43. Raibon E, Todd LM and Moller T: Glial cells in ALS: the missing link? Phys Med Rehabil Clin N Am 19: 441-459, 2008.

44. Mitchell JD and Borasio GD: Amyotrophic lateral sclerosis Lancet 369: 2031-2041, 2007.

45. Rosen DR, Siddique T, Patterson D, et al: Mutations in $\mathrm{Cu} / \mathrm{Zn}$ superoxide dismutase gene are associated with familial amyotrophic lateral sclerosis. Nature 362: 59-62, 1993.
46. Ito $\mathrm{H}$, Wate $\mathrm{R}$, Zhang J, et al: Treatment with edaravone, initiated at symptom onset, slows motor decline and decreases SOD1 deposition in ALS mice. Exp Neurol 213: 448-455, 2008

47. Kabashi E and Durham HD: Failure of protein quality control in amyotrophic lateral sclerosis. Biochim Biophys Acta 1762: 1038-1050, 2006

48. Valentine JS and Hart PJ: Misfolded CuZnSOD and amyotrophic lateral sclerosis. Proc Natl Acad Sci USA 100: 3617-3622, 2003.

49. Yoshino $\mathrm{H}$ and Kimura A: Investigation of the therapeutic effects of edaravone, a free radical scavenger, on amyotrophic lateral sclerosis (Phase II study). Amyotroph Lateral Scler 7: 241-245, 2006.

50. Takahashi R: Edaravone in ALS. Exp Neurol 217: 235-236, 2009.

51. De Lau LM and Breteler MM: Epidemiology of Parkinson's disease. Lancet Neurol 5: 525-535, 2006.

52. Yuan WJ, Yasuhara T, Shingo T, et al: Neuroprotective effects of edaravone-administration on 6-OHDA-treated dopaminergic neurons. BMC Neurosci 9: 75, 2008.

53. Ma L, Cao TT, Kandpal G, et al: Genome-wide microarray analysis of the differential neuroprotective effects of antioxidants in neuroblastoma cells overexpressing the familial Parkinson's disease alpha-synuclein A53T mutation. Neurochem Res 35: 130-142, 2010.

54. Rosati G: The prevalence of multiple sclerosis in the world: an update. Neurol Sci 22: 117-139, 2001.

55. Cohen JA: Emerging therapies for relapsing multiple sclerosis. Arch Neurol 66: 821-828, 2009.

56. Motomura K, Ogura M, Natsume A, Yokoyama $H$ and Wakabayashi T: A free-radical scavenger protects the neural progenitor cells in the dentate subgranular zone of the hippocampus from cell death after X-irradiation. Neurosci Lett 485: 65-70, 2010.

57. Anderson VA, Godber T, Smibert E, Weiskop S and Ekert H: Cognitive and academic outcome following cranial irradiation and chemotherapy in children: a longitudinal study. Br J Cancer 82: 255-262, 2000 .

58. Crossen JR, Garwood D, Glatstein E and Neuwelt EA: Neurobehavioral sequelae of cranial irradiation in adults: a review of radiation-induced encephalopathy. J Clin Oncol 12: 627-642, 1994

59. Monje ML and Palmer T: Radiation injury and neurogenesis. Curr Opin Neurol 16: 129-134, 2003

60. Ishii J, Natsume A, Wakabayashi $\mathrm{T}$, et al: The free-radical scavenger edaravone restores the differentiation of human neural precursor cells after radiation-induced oxidative stress. Neurosci Lett 423: 225-230, 2007. 